\title{
Análise de tendência do índice de precipitação padronizado em Recife-PE
}

\author{
Trend analysis in standardized precipitation index in Recife-PE \\ Análisis de tendencias del índice de precipitación estandarizado en Recife-PE
}

Recebido: 22/06/2021 | Revisado: 29/06/2021 | Aceito: 07/07/2021 | Publicado: 17/07/2021

Vaniele da Silva Barros

ORCID: https://orcid.org/0000-0002-3238-1106

Universidade Federal Rural de Pernambuco, Brasil

E-mail: vaniiellebarros@hotmail.com

Vanessa Karoline Inacio Gomes

ORCID: https://orcid.org/0000-0001-5085-6833

Universidade Federal Rural de Pernambuco, Brasil E-mail: vanessakaroline@outlook.com

Ivanildo Batista da Silva Júnior

ORCID: https://orcid.org/0000-0002-3669-2542

Universidade Federal Rural de Pernambuco, Brasil E-mail: ivanildo.batista13@gmail.com

Anderson Spinelli Valdevino da Silva

ORCID: https://orcid.org/0000-0002-9770-5263

Universidade Federal de Pernambuco, Brasil E-mail: illenips23@gmail.com

Antonio Samuel Alves da Silva

ORCID: https://orcid.org/0000-0002-8759-0036

Universidade Federal Rural de Pernambuco, Brasil

E-mail: samuelmathematical@gmail.com

Lucian Bogdan Bejan

ORCID: https://orcid.org/0000-0002-3292-6065

Universidade Federal Rural de Pernambuco, Brasil

E-mail: lucianbb@gmail.com

Tatijana Stosic

ORCID: https://orcid.org/0000-0002-5691-945X

Universidade Federal Rural de Pernambuco, Brasil E-mail: tastosic@gmail.com

\begin{abstract}
Resumo
O ciclo hidrológico, afetado por mudanças climáticas ocasionadas pelo processo de desenvolvimento e industrialização, tem recebido grande atenção da comunidade científica em todo o mundo, e com isso, muitos estudos foram realizados para mostrar que as mudanças na precipitação estão tornando-se evidentes em escala global. Além disso, os eventos extremos como estiagens prolongadas, inundações e deslizamentos têm causado impactos socioambientais nas grandes áreas urbanas, sendo os alagamentos em superfícies de alta impermeabilização os mais frequentes, devido a deficiência de infraestrutura nos sistemas de drenagem. Por conseguinte, neste trabalho objetivou-se analisar a tendência em séries temporais de Índice de Precipitação Padronizado (SPI) para as escalas de tempo de 1, 3, 6, 12, 24 e 48 meses, utilizando o teste de Mann-Kendall ao nível de significância de 5\%. Para tanto, foram utilizados dados de precipitação mensal da estação meteorológica convencional Recife (Curado), localizada no estado de Pernambuco, Brasil, entre o período de 1962 a 2019. Os resultados apontaram que para as escalas de tempo de 1 e 3 meses não há tendência temporal significativa, enquanto tendências negativas significativas foram obtidas para as demais escalas, o que indicou aumento da severidade de seca. Em relação às frequências de ocorrências, é observado um aumento na ocorrência de períodos secos para condições de longo prazo (SPI-12, SPI-24 e SPI-48) e para os períodos úmidos foi observado aumento apenas na categoria moderadamente úmido.
\end{abstract}

Palavras-chave: Mann-Kendall; Análise de tendências; Índice de precipitação padronizado.

\begin{abstract}
The hydrological cycle, affected by climate change caused by the process of development and industrialization, has received great attention from the scientific community around the world, and with this, many studies have been carried out to show that changes in precipitation are becoming evident on a scale global. In addition, extreme events such as prolonged droughts, floods and landslides have caused social and environmental impacts in large urban areas, with flooding in highly waterproof surfaces being the most frequent ones, due to insufficient infrastructure in drainage systems. Therefore, this work aimed to analyze the trend in time series of the Standardized Precipitation Index (SPI) for the time scales of 1, 3, 6, 12, 24 and 48 months, using the Mann-Kendall test at the level 5\% significance level. For this purpose, monthly precipitation data from the conventional meteorological station Recife (Curado), located in
\end{abstract}


the state of Pernambuco, Brazil, between the period 1962 to 2019 were used. The results showed that for the time scales of 1 and 3 months there is no significant temporal trend, while significant negative trends were obtained for the other scales, which indicated an increase in drought severity. In relation to the frequencies of occurrences, an increase in the occurrence of dry periods is observed for long-term conditions (SPI-12, SPI-24 and SPI-48) and for wet periods an increase was observed only in the moderately humid category.

Keywords: Mann-Kendall; Trend analysis; Standardized precipitation index.

\section{Resumen}

El ciclo hidrológico, afectado por el cambio climático provocado por el proceso de desarrollo e industrialización, ha recibido una gran atención por parte de la comunidad científica de todo el mundo, y con ello se han realizado numerosos estudios que demuestran que los cambios en las precipitaciones se están haciendo evidentes en escala. global. Además, eventos extremos como sequías prolongadas, inundaciones y deslizamientos de tierra han causado impactos sociales y ambientales en grandes áreas urbanas, siendo las inundaciones en superficies altamente impermeables las más frecuentes, debido a la insuficiente infraestructura en los sistemas de drenaje. Por tanto, este trabajo tuvo como objetivo analizar la tendencia en series de tiempo del Índice de Precipitación Estandarizado (SPI) para las escalas de tiempo de 1, 3, 6, 12, 24 y 48 meses, utilizando la prueba de Mann-Kendall al nivel de significancia del 5\%. nivel. Para ello, se utilizaron datos de precipitación mensual de la estación meteorológica convencional Recife (Curado), ubicada en el estado de Pernambuco, Brasil, entre el período 1962 a 2019. Los resultados mostraron que para las escalas de tiempo de 1 y 3 meses hay ninguna tendencia temporal significativa, mientras que se obtuvieron tendencias negativas significativas para las otras escalas, lo que indica un aumento en la severidad de la sequía. En cuanto a la frecuencia de ocurrencias, se observa un aumento en la ocurrencia de períodos secos para condiciones de largo plazo (SPI-12, SPI-24 y SPI-48) y para períodos húmedos se observó un aumento solo en la categoría moderadamente húmeda.

Palabras clave: Mann-Kendall; Análisis de tendencia; Índice de precipitación estandarizado.

\section{Introdução}

O aumento da emissão de gases de efeitos estufa, oriundo principalmente de atividades antropogênicas, vem causando uma preocupação a nível global com o clima. As mudanças climáticas podem ocasionar diversos impactos negativos no meio ambiente e nos seres vivos, pois está intrinsicamente relacionada com o ciclo hidrológico, podendo intensificar os eventos extremos de precipitação, como secas severas e inundações (ANA, 2015). A gravidade e a duração desses eventos têm um efeito direto na disponibilidade de recursos hídricos, o que pode comprometer o desenvolvimento e planejamento de diversos setores, como a produtividade agrícola, segurança alimentar, gestão de recursos hídricos, uso da terra, saúde humana e equilíbrio ecológico (Hlaváčová et al., 2018; Kalantari et al., 2018; Lake, 2003; Patz \& Kovats, 2002).

Nas últimas décadas, o Brasil foi afetado por vários eventos de seca severa e inundações. No Nordeste brasileiro (NEB) as secas são mais recorrentes, pois possui clima predominantemente semiárido caracterizado por uma alta variabilidade sazonal e interanual da precipitação, com episódios extremos de chuva e seca. A variação sazonal está associada à migração e intensidade da Zona de Convergência Intertropical (ZCIT). Durante a estação chuvosa que ocorre em fevereiro-abril, o Atlântico ZCIT atinge sua posição mais ao sul e recobre diretamente o Nordeste do Brasil. A variabilidade interanual com chuvas excessivas e secas severas está relacionada ao El Niño - Oscilação Sul (ENOS) no Oceano Indo-Pacífico equatorial e ao gradiente meridional de Temperatura da Superfície do Mar (TSM) no Atlântico Tropical. A fase positiva do El Niño do ENOS e a fase negativa do gradiente meridional do TSM levam à diminuição das chuvas no Nordeste brasileiro, enquanto durante a fase negativa - La Niña do ENOS e a fase positiva do gradiente meridional do TSM, a taxa de precipitação está acima da média (Robertson, 2004; Marengo et al., 2017). Nos anos acometidos pelos eventos ENOS em que as anomalias preexistentes da TSM são tais que amplificam o impacto direto do ENOS, a região pode sofrer secas extremas (Giannini, 2004) que podem causar graves consequências ambientais, econômicas e sociais (Marengo 2015, Marengo, Torres \& Alves, 2017).

Os eventos extremos como estiagens, inundações e deslizamentos têm causado impactos socioambientais nas grandes áreas urbanas resultando em elevado número de mortes, feridos e desabrigados, perdas econômicas, proliferação de doenças e degradação do meio ambiente (da Silva, 2020). O planejamento urbano é o fator mais importante em esforços para reduzir os impactos das alterações climáticas e o sucesso desse planejamento depende das informações obtidas através de estudos de 
clima urbano, entre quais destacam-se os estudos hidrológicos, tendo em vista que as cidades passaram a ter sérios problemas relacionados as chuvas extremas. De acordo com Zanella (2013), muitas cidades brasileiras têm problemas com alagamentos nas áreas de alta impermeabilização devido a deficiente infraestrutura nos sistemas de drenagem, e com inundações em ambientes localizados próximos aos rios e lagoas, além disso, as cidades localizadas nas planícies costeiras, como é o caso de Recife-PE, são ainda mais vulneráveis, visto que os efeitos das chuvas intensas combinam com maré alta.

Recentemente, Alcântara et al. (2020) avaliaram as mudanças climáticas hidrológicas da cidade de Recife aplicando o teste Mann-Kendal em dados diários das quatro estações localizadas na Região Metropolitana. O estudo (analisando precipitações totais mensais e precipitação máxima diária anual) não detectou as tendências significativas (positivas ou negativas) para a maioria dos dados analisados.

À vista disso, com objetivo de aprofundar o conhecimento das mudanças climáticas hidrológicas em Recife-PE, neste trabalho foram analisadas as tendências nas séries temporais do Índice de precipitação padronizado (Standardized precipitation index - SPI), utilizando o teste estatístico de Mann-Kendall. Vale salientar que o SPI foi introduzido por McKee, Doesken \& Kleist (1993) para avaliar a severidade das condições secas/úmidas e necessita apenas de dados pluviométricos para realizar os cálculos e pode ser comparado entre regiões com condições climáticas distintas (Svoboda, Hayes \& Wood, 2012), inclusive, já foi amplamente aplicado para analisar condições secas/úmidas em diferentes contextos e em diversas partes do mundo (Du et al., 2013; Buttafuoco, Caloiero \& Coscarelli, 2015; Ashraf \& Routray, 2015; Caloiero, 2017; Araújo Júnior et al., 2017; Dos Santos et al., 2017).

\section{Metodologia}

O procedimento aqui realizado para estudar o comportamento da série temporal de interesse tem como base a análise quantitativa, a partir do teste de Mann Kendall e do SPI. Essas técnicas, descritas mais adiante, foram aqui aplicadas com uso do software R Development Core Team (2020).

\subsection{Dados}

Foram utilizadas séries temporais de precipitação diárias entre os anos de 1962 e 2019 da estação climatológica Recife (Curado), localizada nas latitude $-8,05^{\circ}$ sul e longitude $-34,95^{\circ}$ oeste, instalada à $11,3 \mathrm{~m}$ de altura. Os dados foram fornecidos pelo Instituto Nacional de Meteorologia (INMET).

\section{2 Índice de Precipitação Padronizado (SPI)}

O Índice de Precipitação Padronizado foi desenvolvido por McKee, Doesken, e Kleist (1993) para quantificar o déficit de precipitação em múltiplas escalas de tempo (1, 3, 6, 12, 24 e 48 meses) e é recomendado pela Organização Meteorológica Mundial (OMM) para caracterizar a severidade da seca (Hayes et al., 2011). Para o cálculo do SPI, primeiro é necessário ajustar a função densidade de probabilidade (fdp) para o conjunto de dados de precipitação. Dentre as várias distribuições propostas na literatura (Stagge et al., 2005; Svensson, Hannaford \& Prosdocimi, 2017), neste trabalho, foi adotada a distribuição gama, que é amplamente utilizada para ajustar séries temporais de precipitação (McKee, Doesken \& Kleist, 1993). A fdp da gama é dada por

$$
f(x)=\frac{1}{\beta^{\alpha} \Gamma(\alpha)} x^{\alpha-1} e^{-\frac{x}{\beta}}, x>0,
$$


onde $\alpha>0$ é um parâmetro de forma, $\beta>0$ é um parâmetro de escala, $x$ é a quantidade de precipitação e $\Gamma(\alpha)$ é a função gama: $\Gamma(\alpha)=\int_{0}^{\infty} y^{\alpha-1} e^{-y} d y$. Os parâmetros $\alpha$ e $\beta$ são estimados usando o método da máxima verossimilhança, que produz estimativas

$$
\hat{\alpha}=\frac{1}{4 A}\left(1+\sqrt{1+\frac{4 A}{3}}\right), \quad \hat{\beta}=\frac{\bar{x}}{\hat{\alpha}},
$$

onde $\bar{x}$ é o valor médio da quantidade de precipitação, $A \equiv \ln (\bar{x})-\frac{\sum \ln x}{n}$ é usado para fornecer uma representação de fórmula mais compacta, e $n$ é o número de observações. Em seguida, $f(x)$ é integrada em relação a $x$, para obter a probabilidade acumulada

$$
F(x)=\int_{0}^{x} f(x) d x=\frac{1}{\hat{\beta}^{\hat{\alpha}} \Gamma(\hat{\alpha})} \int_{0}^{x} x^{\hat{\alpha}-1} e^{-x / \hat{\beta}} d x .
$$

A função gama é indefinida para $x=0$, e como os dados de precipitação naturalmente contêm zeros, a probabilidade acumulada é considerada da seguinte forma

$$
H(x)=q+(1-q) F(x),
$$

onde $q$ é a probabilidade de precipitação zero, que é calculada como $q=\frac{m}{n}$, onde $m$ representa o número de zeros em uma série de precipitação e $n$ é o número de observações. Por fim, o SPI é gerado pela padronização dos valores obtidos em $H(x)$ (Lloyd-Hughes \& Saunders, 2002). Isto é,

$$
S P I=\left\{\begin{array}{ll}
-\left(t-\frac{c_{0}+c_{1} t+c_{2} t^{2}}{1+d_{1} t+d_{2} t^{2}+d_{3} t^{3}}\right), & 0<H(x)<0,5 \\
+\left(t-\frac{c_{0}+c_{1} t+c_{2} t^{2}}{1+d_{1} t+d_{2} t^{2}+d_{3} t^{3}}\right), & 0,5<H(x) \leq 1,0
\end{array},\right.
$$

com $c_{0}=2,515517 ; c_{1}=0,802853 ; c_{2}=0,010328 ; d_{1}=1,432788 ; d_{2}=0,189269 ; d_{3}=0,001308 ;$ e $t$ dado por

$$
t=\left\{\begin{array}{ll}
\sqrt{\ln \left[\frac{1}{(H(x))^{2}}\right]}, & 0<H(x)<0,5 \\
\sqrt{\ln \left[\frac{1}{(1-H(x))^{2}}\right]}, \quad 0,5<H(x) \leq 1,0
\end{array} .\right.
$$

McKee, Doesken, e Kleist (1993) categorizam o SPI em classes moderadas, severas e extremas para condições secas e úmidas, conforme mostrado na Tabela 1. 
Tabela 1: Categorias secas/úmidas do Índice de precipitação padronizado.

\begin{tabular}{cc}
\hline Categoria & Valores \\
\hline Extremamente seco & SPI $<-2$ \\
Severamente seco & $-2 \leq \mathrm{SPI}<-1,5$ \\
Moderadamente seco & $-1,5 \leq \mathrm{SPI}<-1,0$ \\
Quase normal & $-1,0<\mathrm{SPI}<1,0$ \\
Moderadamente úmido & $1,0 \leq \mathrm{SPI}<1,5$ \\
Severamente úmido & $1,5 \leq \mathrm{SPI}<2,0$ \\
Extremamente úmido & $\mathrm{SPI} \geq 2$ \\
\hline
\end{tabular}

Fonte: McKee et al. (1993).

\subsection{Mann-Kendall}

O teste de Mann-Kendall (Mann, 1945; Kendall, 1975) é um método estatístico não paramétrico (não requer que os dados sigam nenhuma distribuição específica) usado para determinar se uma série temporal tem uma tendência monotônica de crescimento ou decrescimento. É um procedimento baseado em classificação, especialmente adequado para dados que não seguem distribuição normal, dados contendo outliers e tendências não lineares (Birsan et al., 2014). Este teste é baseado na correlação entre os valores das séries temporais e sua ordem temporal, sendo a hipótese nula de que as observações são independentes e distribuídas de forma idêntica (sem tendência), e a hipótese alternativa de que existe uma tendência monotônica (crescente ou decrescente). Para a série temporal $x_{1}, x_{2}, \ldots, x_{n}$ o teste de Mann-Kendall usa a estatística

$$
S=\sum_{i=1}^{n-1} \sum_{j=i+1}^{n} \operatorname{sgn}\left(x_{j}-x_{i}\right)
$$

em que $x_{i}$ e $x_{j}$ são os valores de dados sequenciais, $n$ é o comprimento do conjunto de dados e $\operatorname{sgn}(\cdot)$ é representado por

$$
\operatorname{sgn}\left(x_{j}-x_{i}\right)=\left\{\begin{array}{c}
1, \text { se } x_{j}>x_{i} \\
0, \text { se } x_{j}=x_{i} . \\
-1, \text { se } x_{j}<x_{i}
\end{array}\right.
$$

Partindo do pressuposto de que os dados são independentes e distribuídos de forma idêntica (hipótese nula), a estatística $S$ tem média zero, $E(S)=0$, e variância dada por

$$
\operatorname{Var}(S)=\frac{1}{18}\left[n(n-1)(2 n+5)-\sum_{p=1}^{q} t_{p}\left(t_{p}-1\right)\left(2 t_{p}+5\right)\right],
$$

onde $q$ é o número de grupos vinculados e $t_{p}$ é o número de observações no $p$-ésimo grupo. A estatística de teste $Z$, que tem uma distribuição normal, é calculada com base nos valores $S$ e $\operatorname{Var}(S)$ :

$$
Z=\left\{\begin{array}{cl}
\frac{S-1}{\sqrt{\operatorname{Var}(S)},} & S>0 \\
0, & S=0 . \\
\frac{S+1}{\sqrt{\operatorname{Var}(S)}}, & S<0
\end{array}\right.
$$

Os valores positivos de $Z$ indicam tendências ascendentes, enquanto os valores negativos de $Z$ mostram tendências descendentes. Finalmente, para fins de teste, o $p$-valor $(p v)$ é calculado como

$$
p v=2 \min (\phi(Z), 1-\phi(Z))
$$


onde $\min (\cdot, \cdot)$ representa o mínimo dos dois argumentos e $\phi(\cdot)$ é a função de distribuição cumulativa da distribuição normal padrão - a hipótese nula é rejeitada quando o valor $p$ da estatística padronizada de teste $Z$ é menor que o nível de significância escolhido. Portanto, diz-se que a tendência é decrescente se $Z$ for negativo e crescente se $Z$ for positivo, e o $p V$ for menor que $\alpha$. Neste artigo, foi utilizado o nível de significância $\alpha=0,05$. O teste não paramétrico de Mann-Kendall tem sido amplamente usado para quantificar as tendências em séries de tempo hidro meteorológicas (Nashwan \& Shahid, 2019).

\section{Resultados e Discussão}

A Figura 1 apresenta a evolução temporal mensal da precipitação de acordo com os dados da estação meteorológica Recife (Curado) durante 1962-2019 (Figura 1 (a)), séries SPI (Figura 1 (b)) e distribuição de frequência de SPI (Figura 1 (c)) em diferentes escalas de tempo (SPI-1, SPI-3, SPI-6, SPI-12, SPI-24 e SPI-48). Observa-se os eventos de seca severa nas escalas maiores: SPI-12 (1976, 1983, 1998); SPI-24 e SPI-48 (1999), que estão de acordo com secas históricas na região do Nordeste (Marengo et al., 2017). Estas ocorrências coincidem com episódios de El Niño: 1976-1997, 1982-1983 e 1997-1998 (INPE, 2021). Os eventos chuvosos observados em 1967 (SPI-6, SPI-12), 1968 (SPI-1), 1988 (SPI-24, SPI-48) e 2000 (SPI-3, SPI-6, SPI-12), os quais coincidem com episódios de La Niña: 1967-1968, 1988-1989 e 1999-2000 (INPE, 2021).

Figura 1: (a) Séries mensais de precipitação, (b) Séries de SPI e (c) Distribuição de frequência de séries do SPI em diferentes escalas de tempo (SPI-1, SPI-3, SPI-6, SPI-12, SPI-24 e SPI-48) da estação Recife-Curado.
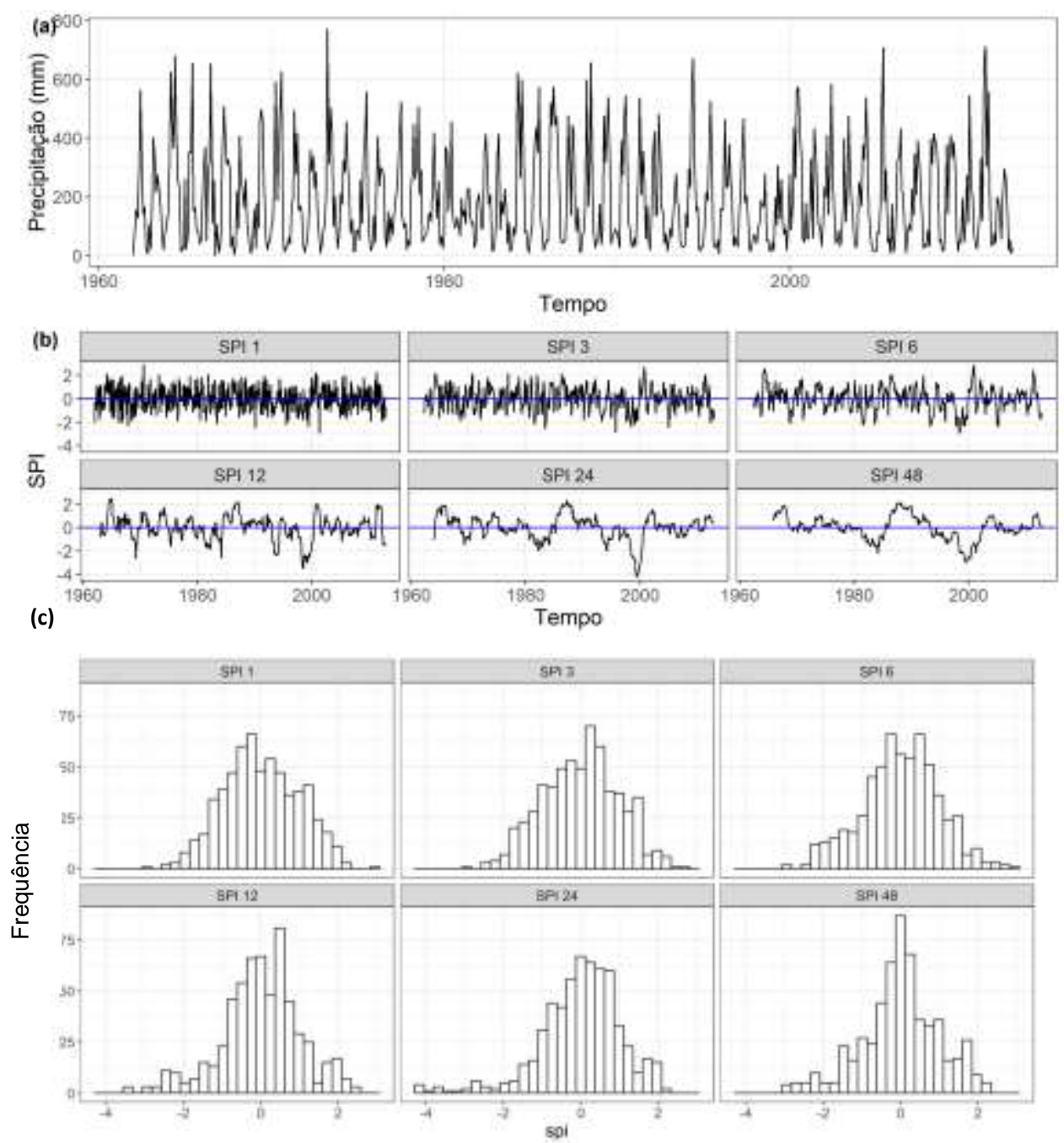

Fonte: Autores (2021). 


\subsection{Análise de tendência}

A tendência monotônica de aumento ou diminuição na precipitação mensal é analisada com o teste não paramétrico de Mann-Kendall (estatística Z). Os resultados da análise de tendência para a série de valores de cada SPI usados neste trabalho estão resumidos na Tabela 2, e apontam que não há tendência temporal de alteração estatisticamente significativa para o SPI-1 e SPI-3. No entanto, os demais SPI apresentaram tendências negativas significativas ao nível de significância confiança de $5 \%$ (p-valor $\leq 0,05$ ). Além disso, nota-se que os valores da estatística $\mathrm{Z}$ diminuem à medida que a escala do SPI aumenta, o que indica uma maior frequência de ocorrência de períodos secos nos últimos anos (Koudahe et al., 2017). De acordo com Azua (2015), a ligeira tendência de aumento e diminuição concorda com o fato de que longas escalas de tempo de SPI respondem lenta e estavelmente à variação na precipitação diária.

Tabela 2: Resultados da análise de tendência para SPI em diferentes escalas de tempo (SPI-1, SPI-3, SPI-6, SPI-12, SPI-24 e SPI-48).

\begin{tabular}{cccc}
\hline SPI & $\mathbf{z}$ & p-valor & Resultado \\
\hline $\mathbf{1}$ & $-0,7934$ & 0,4275 & Sem tendência \\
$\mathbf{3}$ & $-1,7287$ & 0,0839 & Sem tendência \\
$\mathbf{6}$ & $-2,2913$ & 0,0219 & Tendência Negativa \\
$\mathbf{1 2}$ & $-2,7301$ & 0,0063 & Tendência Negativa \\
$\mathbf{2 4}$ & $-3,7974$ & 0,0001 & Tendência Negativa \\
$\mathbf{4 8}$ & $-5,6584$ & 0,0000 & Tendência Negativa \\
\hline
\end{tabular}

Fonte: Autores (2021).

Observa-se que o local estudado experimentou uma tendência de redução da precipitação durante o período de 57 anos deste estudo e se esta tendência continuar, então ela poderia ter repercussões na sustentabilidade dos recursos hídricos superficiais e na recarga das águas subterrâneas, conforme revelado por Green et al. (2011).

\subsection{Probabilidade de ocorrência de diferentes categorias de período úmido e seco}

A probabilidade de ocorrência de várias categorias de períodos úmidos e secos na estação Recife é apresentada nas Tabelas 3, 4, 5 e na Figura 2. Para o SPI-1, a probabilidade de ocorrência de várias categorias de secas e úmidas é mostrada na Tabela 3(a). Os resultados revelaram que 41,4\% do período de estudo foi dominado pela categoria quase normal, apresentando também uma maior probabilidade de ocorrência de 1 em 0,4 ano. Para os períodos secos (27\%) a categoria moderadamente seca apresentou a maior porcentagem e probabilidade de ocorrência, 16,3\% e 1 a cada ano. Para os períodos úmidos (31,7\%) a categoria moderadamente úmida apresentou a maior porcentagem e probabilidade de ocorrência, 18,8\% e 1 a cada 0,8 ano. As categorias extremamente seco e extremamente úmido apresentaram as menores porcentagens de ocorrência, 2,5\% e 2,2\%, e as menores probabilidades, 1 em 6,4 anos e 1 em 7,3 anos, respectivamente. Na análise do SPI-3, retratada na Tabela 3(b), os resultados mostram que o período quase normal apresenta o maior percentual e probabilidade de ocorrência de 37,4\% e 1 a cada 0,6 ano. Isso é seguido por moderadamente seco com porcentagem e probabilidade de ocorrência de 20,6\% e 1 em cada ano. Os períodos extremamente seco e extremamente úmido apresentaram as menores porcentagens, $2,9 \%$ e $2,5 \%$, e probabilidades de ocorrência de 1 em cada 7,3 anos e 1 em cada 8,5 anos, respectivamente. 
Research, Society and Development, v. 10, n. 8, e52310817458, 2021

(CC BY 4.0) | ISSN 2525-3409 | DOI: http://dx.doi.org/10.33448/rsd-v10i8.17458

Tabela 3: Probabilidade de ocorrência de categorias SPI para (a) SPI-1 e (b) SPI-3.

\begin{tabular}{|c|c|c|c|c|}
\hline (a) SPI - 1 & Categoria & $\begin{array}{c}\begin{array}{c}\text { Número de tempo } \\
\text { em } 57 \text { anos }\end{array} \\
\end{array}$ & $\begin{array}{c}\text { Porcentagem de } \\
\text { ocorrência }\end{array}$ & $\begin{array}{c}\text { Severidade dos } \\
\text { eventos }\end{array}$ \\
\hline$<-2$ & Extremamente seco & 8 & $2,5 \%$ & 1 em 6,4 anos \\
\hline$-2 \mathrm{a}-1,5$ & Severamente seco & 26 & $8,2 \%$ & $1 \mathrm{em} 2$ anos \\
\hline$-1,5$ a -1 & Moderadamente seco & 52 & $16,3 \%$ & 1 em 1 anos \\
\hline-1 a 1 & Quase normal & 132 & $41,4 \%$ & 1 em 0,4 anos \\
\hline 1 a 1,5 & Moderadamente úmido & 60 & $18,8 \%$ & 1 em 0,8 anos \\
\hline 1,5 a 2 & Severamente úmido & 34 & $10,7 \%$ & 1 em 1,5 anos \\
\hline$\geq 2$ & Extremamente úmido & 7 & $2,2 \%$ & $1 \mathrm{em} 7,3$ anos \\
\hline (b) SPI - 3 & Categoria & $\begin{array}{c}\text { Número de tempo } \\
\text { em } 57 \text { anos }\end{array}$ & $\begin{array}{c}\text { Porcentagem de } \\
\text { ocorrência }\end{array}$ & $\begin{array}{c}\begin{array}{c}\text { Severidade dos } \\
\text { eventos }\end{array} \\
\end{array}$ \\
\hline$<-2$ & Extremamente seco & 7 & $2,9 \%$ & $1 \mathrm{em} 7,3$ anos \\
\hline$-2 \mathrm{a}-1,5$ & Severamente seco & 23 & $9,5 \%$ & 1 em 2,2 anos \\
\hline$-1,5$ a -1 & Moderadamente seco & 50 & $20,6 \%$ & 1 em 1 anos \\
\hline-1 a 1 & Quase normal & 91 & $37,4 \%$ & 1 em 0,6 anos \\
\hline 1 a 1,5 & Moderadamente úmido & 46 & $18,9 \%$ & $1 \mathrm{em} 1,1$ anos \\
\hline 1,5 a 2 & Severamente úmido & 20 & $8,2 \%$ & $1 \mathrm{em} 2,5$ anos \\
\hline$\geq 2$ & Extremamente úmido & 6 & $2,5 \%$ & $1 \mathrm{em} 8,5$ anos \\
\hline
\end{tabular}

Fonte: Autores (2021).

Na análise do SPI-6 e do SPI-12, os resultados, expostos na Tabela 4, revelaram que a categoria quase normal apresentou a maior porcentagem e probabilidade de ocorrência. Ainda para o SPI-6, observa-se que os períodos úmidos obtiveram 31,6\% de ocorrência, e os eventos extremamente úmidos, especificamente, apresentaram a menor porcentagem $(2,8 \%)$ e probabilidade de ocorrência de 1 em 10,2 anos. Enquanto os períodos secos totalizaram 33,8\% com intensidades variadas. O SPI-12 revelou períodos secos (37,4\%), seguido por períodos úmidos (33\%), com diferentes intensidades, e verificou-se que os eventos extremamente secos e extremamente úmidos apresentaram a mesma porcentagem e probabilidade de ocorrência de 4,4\% e 1 em cada 12,8 anos. A persistência de períodos de seca requer atenção, visto que, os eventos menos intensos de secas podem se desenvolver para mais intensos, de acordo com à atuação de fenômenos externos (Nascimento, Braga \& Araújo, 2017), o que pode contribuir com agravamentos nos setores hídricos. 
Research, Society and Development, v. 10, n. 8, e52310817458, 2021

(CC BY 4.0) | ISSN 2525-3409 | DOI: http://dx.doi.org/10.33448/rsd-v10i8.17458

Tabela 4: Probabilidade de ocorrência de categorias SPI para (a) SPI-6 e (b) SPI-12.

\begin{tabular}{|c|c|c|c|c|}
\hline (a) SPI - 6 & Categoria & $\begin{array}{c}\text { Número de tempo } \\
\text { em } 57 \text { anos }\end{array}$ & $\begin{array}{c}\text { Porcentagem de } \\
\text { ocorrência }\end{array}$ & $\begin{array}{c}\text { Severidade dos } \\
\text { eventos }\end{array}$ \\
\hline$<-2$ & Extremamente seco & 11 & $6,2 \%$ & 1 em 4,6 anos \\
\hline$-2 \mathrm{a}-1,5$ & Severamente seco & 16 & $9,0 \%$ & 1 em 3,2 anos \\
\hline$-1,5$ a -1 & Moderadamente seco & 33 & $18,6 \%$ & $1 \mathrm{em} 1,5$ anos \\
\hline-1 a 1 & Quase normal & 61 & $34,5 \%$ & 1 em 0,8 anos \\
\hline 1 a 1,5 & Moderadamente úmido & 36 & $20,3 \%$ & $1 \mathrm{em} 1,4$ anos \\
\hline 1,5 a 2 & Severamente úmido & 15 & $8,5 \%$ & 1 em 3,4 anos \\
\hline$\geq 2$ & Extremamente úmido & 5 & $2,8 \%$ & $1 \mathrm{em} 10,2$ anos \\
\hline (b) SPI - 12 & Categoria & $\begin{array}{c}\begin{array}{c}\text { Número de tempo } \\
\text { em } 57 \text { anos }\end{array} \\
\end{array}$ & $\begin{array}{c}\text { Porcentagem de } \\
\text { ocorrência }\end{array}$ & $\begin{array}{c}\text { Severidade dos } \\
\text { eventos }\end{array}$ \\
\hline$<-2$ & Extremamente seco & 4 & $4,4 \%$ & 1 em 12,8 anos \\
\hline-2 a $-1,5$ & Severamente seco & 11 & $12,1 \%$ & $1 \mathrm{em} 4,6$ anos \\
\hline$-1,5$ a -1 & Moderadamente seco & 19 & $20,9 \%$ & $1 \mathrm{em} 2,7$ anos \\
\hline-1 a 1 & Quase normal & 27 & $29,7 \%$ & 1 em 1,9 anos \\
\hline 1 a 1,5 & Moderadamente úmido & 18 & $19,8 \%$ & $1 \mathrm{em} 2,8$ anos \\
\hline 1,5 a 2 & Severamente úmido & 8 & $8,8 \%$ & 1 em 6,4 anos \\
\hline$\geq 2$ & Extremamente úmido & 4 & $4,4 \%$ & $1 \mathrm{em} 12,8$ anos \\
\hline
\end{tabular}

Fonte: Autores (2021).

Com relação às análises dos SPI-24 e SPI-48, apresentadas na Tabela 5, assim como para os SPI anteriores, a categoria quase normal apresentou a maior porcentagem e probabilidade de ocorrência. O SPI-24 mostra que 35,1\% do período de estudo são secos e 33,3\% são úmidos, ambos com intensidades variadas. A categoria extremamente seca apresentou a menor porcentagem e probabilidade de ocorrência de 1,7\% e 1 em cada 51 anos, seguido por extremamente úmido com ocorrência de 3,3\% e 1 em cada 25,5 anos. Os resultados para o SPI-48, apresentados na Tabela 5(b), revelaram que 35,9\% do intervalo de tempo estudado, apresentou períodos secos com intensidades variadas. Para os períodos úmidos (33,9\%), destacase a categoria extremamente úmido, apresentando a menor porcentagem e probabilidade de ocorrência de $1,9 \%$ e 1 em cada 51 anos. 
Tabela 5: Probabilidade de ocorrência de categorias SPI para (a) SPI-24 e (b) SPI-48.

\begin{tabular}{|c|c|c|c|c|}
\hline (a) SPI - 24 & Categoria & $\begin{array}{c}\text { Número de tempo } \\
\text { em } 57 \text { anos }\end{array}$ & $\begin{array}{c}\text { Porcentagem de } \\
\text { ocorrência }\end{array}$ & $\begin{array}{c}\text { Severidade dos } \\
\text { eventos }\end{array}$ \\
\hline$<-2$ & Extremamente seco & 1 & $1,7 \%$ & 1 em 51 anos \\
\hline-2 a $-1,5$ & Severamente seco & 4 & $6,7 \%$ & $1 \mathrm{em} 12,8$ anos \\
\hline$-1,5 \mathrm{a}-1$ & Moderadamente seco & 16 & $26,7 \%$ & $1 \mathrm{em} 3,2$ anos \\
\hline-1 a 1 & Quase normal & 19 & $31,7 \%$ & $1 \mathrm{em} 2,7$ anos \\
\hline 1 a 1,5 & Moderadamente úmido & 11 & $18,3 \%$ & 1 em 4,6 anos \\
\hline 1,5 a 2 & Severamente úmido & 7 & $11,7 \%$ & $1 \mathrm{em} 7,3$ anos \\
\hline$\geq 2$ & Extremamente úmido & 2 & $3,3 \%$ & $1 \mathrm{em} 25,5$ anos \\
\hline (b) SPI - 48 & Categoria & $\begin{array}{c}\text { Número de tempo } \\
\text { em } 57 \text { anos }\end{array}$ & $\begin{array}{c}\text { Porcentagem de } \\
\text { ocorrência }\end{array}$ & $\begin{array}{c}\text { Severidade dos } \\
\text { eventos }\end{array}$ \\
\hline$<-2$ & Extremamente seco & 3 & $5,7 \%$ & 1 em 17 anos \\
\hline-2 a $-1,5$ & Severamente seco & 7 & $13,2 \%$ & $1 \mathrm{em} 7,3$ anos \\
\hline$-1,5$ a -1 & Moderadamente seco & 9 & $17,0 \%$ & $1 \mathrm{em} 5,7$ anos \\
\hline-1 a 1 & Quase normal & 16 & $30,2 \%$ & 1 em 3,2 anos \\
\hline 1 a 1,5 & Moderadamente úmido & 13 & $24,5 \%$ & 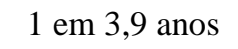 \\
\hline 1,5 a 2 & Severamente úmido & 4 & $7,5 \%$ & 1 em 12,8 anos \\
\hline$\geq 2$ & Extremamente úmido & 1 & $1,9 \%$ & $1 \mathrm{em} 51$ anos \\
\hline
\end{tabular}

Fonte: Autores (2021).

Verificou-se que para as escalas de longo prazo (SPI-12, SPI-24 e SPI-48), apresentou um destaque para os períodos secos, refletindo a clima de Nordeste brasileiro caracterizada com secas frequentes. O clima em todas as análises é quase normal de acordo com a classificação de SPI de McKee (1993). Os resultados mostram que condições de seca severa (-2,00 $\leq$ SPI $<-1,50)$ a extrema seca (SPI < -2,00) ocorreram para os seis SPI estudados em uma média de intervalos de tempo de 2 a 51 anos. Além disso, foi observado que condições de seca moderada $(-1,50 \leq \mathrm{SPI}<-1,00)$ ocorreram em uma média de 1 a 5,7 anos e porcentagens de ocorrência de $16,3 \%$ a $26,7 \%$.

Recentemente da Silva et al. (2020) analisaram casos/anos de eventos secos e chuvosos no período de 1961 a 2014 , para o leste do Nordeste do Brasil através do índice SPI-1 e na distribuição de frequência do SPI, verificaram que 38,42\% dos eventos ocorreram na categoria de seca e 27,32 \% dos eventos na categoria úmida. Os resultados da análise para um período maior (1962-2019) mostraram para SPI-1 27\% dos eventos na categoria de seca (extrema, severa e moderada) e $31.6 \%$ dos eventos na categoria úmida. Além do SPI-1, a análise inclui SPI em outras escalas temporais $(3,6$,9 ,12 e 48) e assim fornece uma informação mais detalhada sobre as tendências das condições climáticas (secos e chuvosos) na localização estudada: i) SPI-1 reflete as condições de curto prazo (pode ser usado como um indicador para impactos imediatos como redução da umidade do solo que é importante para agricultura) e não mostraram tendência (Svoboda, Hayes \& Wood, 2012); ii) O SPI-3 e SPI-6 indicam tendências sazonais a médio prazo na precipitação (pode estar associado a fluxos anômalos e níveis de reservatórios), SPI-12, SPI-24 e SPI-48 que pode ser usado como um indicador para redução da recarga do reservatório e da água subterrânea, todos mostram tendência negativa (Svoboda, Hayes \& Wood, 2012).

O histograma de frequências das 7 categorias do SPI 1, 3, 6, 12, 24 e 48 meses é ilustrado na Figura 2. De modo geral, observa-se que a frequência dos eventos secos/úmidos é inversamente proporcional à escala de tempo do SPI, isto é, à medida que a escala de tempo do SPI aumenta a frequência de eventos secos/chuvosos diminui (Dos Santos et al., 2014). 
Figura 2: Distribuição da probabilidade de ocorrência das categorias do SPI em diferentes escalas de tempo (SPI-1, SPI-3, SPI-6, SPI-12, SPI-24 e SPI-48).

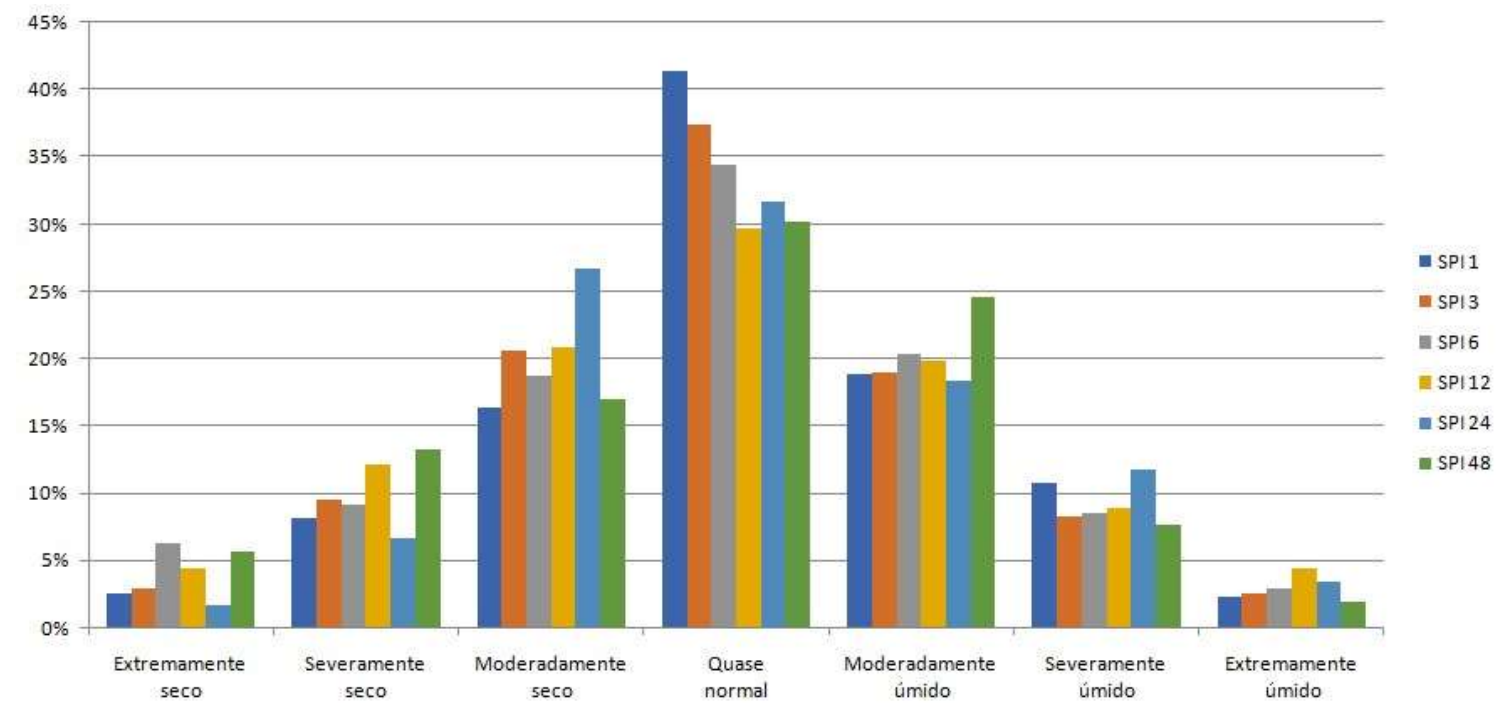

Fonte: Autores (2021).

\section{Considerações Finais}

A análise das séries temporais do SPI em diferentes escalas de tempo e a frequência de ocorrência de períodos secos, normais e com umidade, mostram que: (i) para as escalas de tempo 1 e 3 do SPI, não foram identificadas tendências estatisticamente significativas, porém a partir da escala de tempo $6 \mathrm{em}$ diante a estatística z calculada mostrou-se negativa e estatisticamente significativa ( $p$-valor $\leq 0,05$ ), indicando que para as escalas de médio e longo prazo há uma tendência de aumento de severidade de seca. As frequências de ocorrências indicam que: (ii) À medida que a escala do índice SPI para a estação Recife aumenta, a porcentagem de probabilidade de ocorrência de períodos quase normais diminui (de 0,413 no SPI 1 e 0,302 no SPI 48); (iii) entretanto, a porcentagem de probabilidade de ocorrência de períodos secos (extremos, severos e moderados) aumenta; e para os períodos úmidos, o único aumento identificado foi em períodos do tipo moderadamente úmidos.

O presente trabalho representa uma análise compreensiva das condições climáticas em Recife a partir de uma série histórica de precipitação. Os resultados podem ser úteis para planejamento urbano, para reduzir os impactos das alterações climáticas. Trabalhos futuros podem incluir mais estações pluviométricas que permitirão uma análise espacial de tendências do SPI e determinarão quais regiões sofreram com mudanças significativas ao longo do tempo.

\section{Agradecimentos}

Os autores agradecem ao CNPq (Conselho Nacional de Desenvolvimento Científico e Tecnológico), à CAPES (Coordenação de Aperfeiçoamento de Pessoal de Nível Superior), à FACEPE (Fundação de Amparo à Ciência e Tecnologia do Estado de Pernambuco) e ao Instituto Nacional de Meteorologia (INMET) pelo apoio ao desenvolvimento desta pesquisa.

\section{Referências}

Agência Nacional de Águas (Brasil). Conjuntura dos recursos hídricos no Brasil: regiões hidrográficas brasileiras - Edição Especial. ANA, 2015.

Araújo Júnior, L. M. D., Souza Filho, F. D. A., Silveira, C. D. S., Dias, T. A., \& Doss-Gollin, J. (2014). Análise dos eventos de seca no nordeste setentrional brasileiro com base no índice de precipitação normalizada. XII Simpósio de Recursos Hídricos do Nordeste. 
Ashraf, M., \& Routray, J. K. (2015). Spatio-temporal characteristics of precipitation and drought in Balochistan Province, Pakistan. Natural Hazards, 77(1), 229-254.

Azua, S. (2015). Analysis of Rainfall Variability and the Trends of Wet and Dry Periods in Makurdi and Environs Using Standardised Precipitation Index. In 6th International Conference and Annual General Meeting Meeting of Nigeria Association of Hydrological Sciences (NAHS) "ABU (pp. 1-11).

Birsan, M. V., Dumitrescu, A., Micu, D. M., \& Cheval, S. (2014). Changes in annual temperature extremes in the Carpathians since AD 1961. Natural Hazards, 74(3), 1899-1910.

Buttafuoco, G., Caloiero, T., \& Coscarelli, R. (2015). Analyses of drought events in Calabria (southern Italy) using standardized precipitation index. Water Resources Management, 29(2), 557-573.

Caloiero, T. (2017). Drought analysis in New Zealand using the standardized precipitation index. Environmental Earth Sciences, 76(16), 1-13.

Chadwick, R., Good, P., Martin, G., \& Rowell, D. P. (2016). Large rainfall changes consistently projected over substantial areas of tropical land. Nature Climate Change, 6(2), 177-181.

Da Silva, D. F., Lima, M. J. S., Souza Neto, P. F., Gomes, H. B., Silva, F. D. S., Almeida, H. R. R. C., \& Pereira, M. P. S. (2020). Caracterização de eventos extremos e de suas causas climáticas com base no Índice Padronizado de Precipitação Para o Leste do Nordeste. Revista Brasileira de Geografia Física, 13(02), 449-464.

De Alcântara, L. R. P., da Silva, M. E. R., dos Santos Neto, S. M., Lafayette, F. B., Coutinho, A. P., Montenegro, S. M. G. L., \& Antonino, A. C. D. (2020). Mudanças climáticas e tendências do regime pluviométrico do Recife. Research, Society and Development, 9(3), e178932583-e178932583.

Debortoli, N. S., Camarinha, P. I. M., Marengo, J. A., \& Rodrigues, R. R. (2017). An index of Brazil's vulnerability to expected increases in natural flash flooding and landslide disasters in the context of climate change. Natural hazards, 86(2), 557-582.

De Sousa Oliveira, G. C., da Silva Junior, J. P., Nóbrega, R. S., \& Girão, O. (2011). Uma Abordagem da Geografia do Clima Sobre os Eventos Extremos de Precipitação em Recife-PE (An Climate Geography Approach on Extreme Precipitation Events in Recife-PE). Revista Brasileira de Geografia Física, 4(2), 238-251.

Dos Santos, S. R. Q., Braga, C. C., Santos, A. P. P., Brito, J. I. B., \& Campos, T. L. O. B. (2014). Classificação de eventos extremos de precipitação em múltiplas escalas de tempo em Belém-PA: Utilizando o índice de precipitação normalizada. Revista Brasileira de Geografia Física, 7(4), 628-635.

Dos Santos, S. R. Q. D., Braga, C. C., Sansigolo, C. A., \& Santos, A. P. P. D. (2017). Determinação de regiões homogêneas do índice de precipitação normalizada (SPI) na Amazônia Oriental. Revista Brasileira de Meteorologia, 32, 111-122.

Du, J., Fang, J., Xu, W., \& Shi, P. (2013). Analysis of dry/wet conditions using the standardized precipitation index and its potential usefulness for drought/flood monitoring in Hunan Province, China. Stochastic environmental research and risk assessment, 27(2), 377-387.

Giannini, A., Saravanan, R., \& Chang, P. (2004). The preconditioning role of tropical Atlantic variability in the development of the ENSO teleconnection: Implications for the prediction of Nordeste rainfall. Climate Dynamics, 22(8), 839-855.

Green, T. R., Taniguchi, M., Kooi, H., Gurdak, J. J., Allen, D. M., Hiscock, K. M., \& Aureli, A. (2011). Beneath the surface of global change: Impacts of climate change on groundwater. Journal of Hydrology, 405(3-4), 532-560.

Hayes, M., Svoboda, M., Wall, N., \& Widhalm, M. (2011). The Lincoln declaration on drought indices: universal meteorological drought index recommended. Bulletin of the American Meteorological Society, 92(4), 485-488.

Hlaváčová, M., Klem, K., Rapantová, B., Novotná, K., Urban, O., Hlavinka, P., \& Trnka, M. (2018). Interactive effects of high temperature and drought stress during stem elongation, anthesis and early grain filling on the yield formation and photosynthesis of winter wheat. Field crops research, 221, 182-195.

Kalantari, Z., Ferreira, C. S. S., Keesstra, S., \& Destouni, G. (2018). Nature-based solutions for flood-drought risk mitigation in vulnerable urbanizing parts of East-Africa. Current Opinion in Environmental Science \& Health, 5, 73-78.

Kendall, M. G. (1948). Rank correlation methods. Griffin.

Koudahe, K., Kayode, A. J., Samson, A. O., Adebola, A. A., \& Djaman, K. (2017). Trend analysis in standardized precipitation index and standardized anomaly index in the context of climate change in Southern Togo. Atmospheric and Climate Sciences, 7(04), 401.

El Niño e La Niña - CPTEC/INPE. (2021). Inpe.br. http://enos.cptec.inpe.br/

Lake, P. S. (2003). Ecological effects of perturbation by drought in flowing waters. Freshwater biology, 48(7), 1161-1172.

Lloyd-Hughes, B., \& Saunders, M. A. (2002). A drought climatology for Europe. International Journal of Climatology: A Journal of the Royal Meteorological Society, 22(13), 1571-1592.

Mann, H. B. (1945). Nonparametric tests against trend. Econometrica: Journal of the econometric society, 245-259.

Marengo, J. A., Torres, R. R., \& Alves, L. M. (2017). Drought in Northeast Brazil—past, present, and future. Theoretical and Applied Climatology, 129(3), $1189-1200$

Marengo, J. A., Alves, LM, Alvala, R., Cunha, AP, Brito, S., \& Moraes, OL (2017). Características climáticas da seca de 2010-2016 na região semiárida do Nordeste do Brasil. Anais da Academia Brasileira de Ciências, 90, 1973-1985.

Marengo, J. A., \& Bernasconi, M. (2015). Regional differences in aridity/drought conditions over Northeast Brazil: present state and future projections. Climatic Change, 129(1), 103-115. 
Research, Society and Development, v. 10, n. 8, e52310817458, 2021

(CC BY 4.0) | ISSN 2525-3409 | DOI: http://dx.doi.org/10.33448/rsd-v10i8.17458

McKee, T. B., Doesken, N. J., \& Kleist, J. (1993, January). The relationship of drought frequency and duration to time scales. In Proceedings of the 8th Conference on Applied Climatology (Vol. 17, No. 22, pp. 179-183).

Nascimento, F. C. A., Braga, C. C., Araújo, F. R. C. D. 2017. Análise Estatística dos Eventos Secos e Chuvosos de Precipitação do Estado do Maranhão. Revista Brasileira de Meteorologia, 32, 375-386.

Nashwan, M. S., \& Shahid, S. (2019). Spatial distribution of unidirectional trends in climate and weather extremes in Nile river basin. Theoretical and applied climatology, 137(1), 1181-1199.

Palmer, W. C. (1968). Keeping track of crop moisture conditions, nationwide: the new crop moisture index. Weatherwise, 21, 156-161.

Patz, J. A., \& Kovats, R. S. (2002). Hotspots in climate change and human health. Bmj, 325(7372), 1094-1098.

R CORE TEAM (2020). R: A language and environment for statistical computing. R Foundation for Statistical Computing, Vienna, Austria. URL: http://www.R-project.org/.

Robertson, A. W., Kirshner, S., \& Smyth, P. (2004). Downscaling of daily rainfall occurrence over northeast Brazil using a hidden Markov model. Journal of climate, $17(22), 4407-4424$

Shukla, S., \& Wood, A. W. (2008). Use of a standardized runoff index for characterizing hydrologic drought. Geophysical research letters, 35(2).

Stagge, J. H., Tallaksen, L. M., Gudmundsson, L., Van Loon, A. F., \& Stahl, K. (2015). Candidate distributions for climatological drought indices (SPI and SPEI). International Journal of Climatology, 35(13), 4027-4040.

Svensson, C., Hannaford, J., \& Prosdocimi, I. (2017). Statistical distributions for monthly aggregations of precipitation and streamflow in drought indicator applications. Water Resources Research, 53(2), 999-1018.

Svoboda, M., Hayes, M., \& Wood, D. (2012). Standardized precipitation index user guide. World Meteorological Organization Geneva, Switzerland, 900.

Vicente-Serrano, S. M., Beguería, S., \& López-Moreno, J. I. (2010). A multiscalar drought index sensitive to global warming: the standardized precipitation evapotranspiration index. Journal of climate, 23(7), 1696-1718.

Zanella, M. E., \& de Oliveira Moura, M. (2013). O clima das cidades do Nordeste brasileiro: contribuições no planejamento e gestão urbana. Revista da ANPEGE, 9(11), 75-89. 